\title{
КОММУТАТИВНЫЕ ИДЕАЛЫ НАИБОЛЬШЕЙ РАЗМЕРНОСТИ НИЛЬТРЕУГОЛЬНОЙ ПОДАЛГЕБРЫ АЛГЕБРЫ ШЕВАЛЛЕ НАД ПОЛЕМ
}

\author{
Е. А. Кириллова, Г. С. Сулейманова
}

\begin{abstract}
Пусть $N$ - нильтреугольная подалгебра алгебры Шевалле. В статье изучается проблема описания коммутативных подалгебр наибольшей размерности подалгебры $N$ над произвольным полем. Доказывается, что $N$ содержит коммутативный идеал этой размерности. Найдены все такие идеалы. Также описаны максимальные коммутативные идеалы подалгебры $N$ для типов $G_{2}$ и $F_{4}$. Как следствие, найдена наибольшая размерность коммутативных подалгебр во всех подалгебрах $N$.

Ключевые слова: алгебра Шевалле, нильтреугольная подалгебра, коммутативные идеалы и идеалы наибольшей размерности.
\end{abstract}

E. A. Kirillova, G.S. Suleimanova. Highest dimension commutative ideals of a niltriangular subalgebra of a Chevalley algebra over a field.

Let $N$ be a niltriangular subalgebra of a Chevalley algebra. We study the problem of describing commutative ideals of $N$ of the highest dimension over an arbitrary field. It is proved that $N$ contains a commutative ideal of this dimension, and all such ideals are found. In addition, all maximal commutative ideals of $N$ are described for the types $G_{2}$ and $F_{4}$. As a consequence, the highest dimension of commutative subalgebras in all subalgebras of $N$ is found.

Keywords: Chevalley algebra, niltriangular subalgebra, commutative ideals and highest dimension ideals.

MSC: 17B05, 17B30

DOI: $10.21538 / 0134-4889-2018-24-3-98-108$

\section{Введение}

И. Шур [1] указал наибольшую размерность абелевых подгрупп группы $S L(n, \mathbb{C})$ и доказал, что абелевы подгруппы этой размерности при $n>3$ автоморфны. В 1945 г. А. И. Мальцев [2] исследовал задачу описания абелевых подгрупп максимальной размерности во всех конечномерных комплексных простых группах Ли, применяя переход к комплексным алгебрам Ли.

Методы [2] модифицировались и применялись к проблеме описания больших абелевых подгрупп конечной группы Шевалле и редукционной задаче для унипотентного радикала $U$ ее подгруппы Бореля, [3-10]. Большой $\mathcal{P}$-nодгруппой конечной группы (P - любое теоретикогрупповое свойство) называют все ее $\mathcal{P}$-подгруппы наибольшего порядка.

Пусть $L_{\Phi}(K)$ - алгебра Шевалле над произвольным полем $K$, ассоциированная с системой корней $\Phi[11, \S 4.4]$. Базу Шевалле в ней составляют элементы $e_{r}(r \in \Phi)$ и подходящая база подалгебры Картана. Элементы $e_{r}\left(r \in \Phi^{+}\right)$образуют базу нильтреугольной подалгебры $N \Phi(K)$ алгебры $L_{\Phi}(K)$. Свою задачу А. И. Мальцев решил редукцией к аналогичной задаче для алгебр Ли $N \Phi(\mathbb{C})$.

В [12] наряду с обобщенной задачей Мальцева записана следующая задача.

Обобщенная редукционная задача. Описать коммутативные подалгебры наибольшей размерности в подалгебре $N \Phi(K)$ алгебры Шевалле $L_{\Phi}(K)$ над произвольным полем $K$.

\footnotetext{
${ }^{1}$ Работа выполнена при поддержке РФФИ (проект 16-01-00707).
} 
Разработка в [13] решения этой задачи развивает схему [9], использующую методы А. И. Мальцева и Е.П. Вдовина. В настоящей статье (теорема 7) подтверждается высказанная в [13] гипотеза.

Гипотеза (A). Всякий коммутативный идеал наибольшей размерности алгебры $N \Phi(K)$ является ее коммутативной подалгеброй наибольшей размерности.

Вместе с тем для алгебры Ли $N \Phi(K)$ указаны явно наибольшие размерности коммутативных подалгебр и завершено описание коммутативных идеалов наибольшей размерности (табл. 1 и 2).

\section{1. Предварительные замечания и тип $G_{2}$}

Алгебру Шевалле $L_{\Phi}(K)$ над полем $K$, ассоциированную с системой корней $\Phi$ евклидова пространства, характеризуют $[11, \S 4.4]$ базой Шевалле, состоящей из элементов $e_{r}(r \in \Phi)$ вместе с подходящей базой подалгебры Картана. Элементы $e_{r}\left(r \in \Phi^{+}\right)$образуют базу максимальной нильпотентной подалгебры $N \Phi(K)$. По теореме Шевалле о базисе если $r, s \in \Phi^{+}$, то

$$
e_{r} * e_{s}=N_{r s} e_{r+s}, \quad N_{s r}=-N_{r s} \quad(r+s \in \Phi), \quad e_{r} * e_{s}=0 \quad(r+s \notin \Phi),
$$

где $N_{r s}= \pm 1$, или $|r|=|s|<|r+s|$ и $N_{r s}= \pm 2$, или $\Phi$ типа $G_{2}$ и $N_{r s}= \pm 2$ или \pm 3 .

Сумму $\mathrm{ht}(r)$ коэффициентов в разложении корня $r$ по базису называют высотой корня. В алгебре $N \Phi(K)$ выделяют идеалы $L_{i}=\left\langle K e_{r} \mid r \in \Phi^{+}, \operatorname{ht}(r) \geqslant i\right\rangle$. Через $\{r\}^{+}$обозначаем множество корней $s \in \Phi^{+}$таких, что в разложении $s-r$ по базису все коэффициенты неотрицательны, через $T(r)$ и $Q(r)$ - подалгебры в $N \Phi(K)$ с базисами $\left\{e_{s} \mid s \in\{r\}^{+}\right\}$и $\left\{e_{s} \mid s \in\{r\}^{+} \backslash\{r\}\right\}$ соответственно. Если $H \subseteq T\left(r_{1}\right)+T\left(r_{2}\right)+\cdots+T\left(r_{m}\right)$ и включение нарушается при любой замене $T\left(r_{i}\right)$ на $Q\left(r_{i}\right)$, то назовем $\left\{r_{1}, r_{2}, \cdots, r_{m}\right\}=\mathcal{L}(H)$ множеством углов для $H$.

Описание коммутативных идеалов наибольшей размерности в алгебре Ли $N \Phi(K)$ над произвольным полем $K$ естественно получать, по аналогии с [9], из решения более общей задачи:

(Б) Описать максимальные коммутативные идеалы алгебры Ли $N \Phi(K)$ над полем $K$.

Решение задачи (Б) для типа $G_{2}$, а при $2 K=K$ также для типа $F_{4}$ анонсировалось ранее в [14]. Мы используем представление из [15, табл. IX] системы корней типа $G_{2}$ с базой $\{a, b\}$, где $|a|<|b|$.

Теорема 1. Все максимальные коммутативные идеалы алгебры Ли $N \Phi(K)$ mипа $G_{2}$ исчерпывают при $6 K=K$ идеал $L_{3}$, при $3 K=0$ идеаль $L_{2} u$

$$
K\left(e_{b}+d e_{b+3 a}\right)+K e_{b+a}+K e_{b+2 a}+L_{5} \quad(d \in K),
$$

наконеи, при $2 K=0$ идеаль $L_{3}, K e_{a}+K e_{b+a}+L_{4}, K\left(e_{b+a}+d e_{b+2 a}\right)+L_{4}(d \in K, d \neq 0) u$

$$
K\left(e_{b}+c e_{2 a+b}+d e_{b+3 a}\right)+K\left(e_{a+b}+c e_{b+3 a}\right)+L_{5} \quad(c, d \in K) .
$$

Д о к а з а т е л ь с т в о. Исследуем произвольный максимальный коммутативный идеал $M$ в $N \Phi(K)$. Он обязан совпадать со своим централизатором $C(M)=\{\beta \in N \Phi(K) \mid \beta * M=0\}$.

Предположим, $M \nsubseteq L_{3}$. Тогда в $M$ существует элемент $\alpha$ с углом $a+b$ :

$$
\alpha=\sum_{r \in \Phi^{+}} c_{r} e_{r} \in M, \quad c_{a}=c_{b}=0, \quad c_{a+b} \neq 0 .
$$

Если $M$ имеет простой угол, то такой элемент можно выбрать в $M * e_{b}$ или в $M * e_{a}$. Учитывая коммутативность идеала $M$, приходим к равенствам

$$
0=\left(e_{a} * \alpha\right) * \alpha= \pm 2 c_{b+a}\left(e_{b+2 a} * \alpha\right)= \pm 6 c_{b+a}^{2} e_{b+3 a} \quad \bmod L_{5} .
$$


При $6 K=K$ это дает противоречие с условием $c_{a+b} \neq 0$. Поэтому здесь $M=L_{3}$.

Пусть $3 K=0$. Тогда $L_{2}-$ коммутативный идеал. Кроме того, $K e_{b+2 a}+L_{5}$ есть центр в $N G_{2}(K)$, и поэтому он лежит в $M$. Если $M$ имеет простой угол $a$, то произведение $M * \alpha$ имеет угол $2 a+b$, т. е. является ненулевым; это противоречит коммутативности $M$. Следовательно, $M \subseteq T(b)$ и централизатор $C(M)=M$ содержит централизатор $C(T(b))=K e_{a+b}+K e_{2 a+b}+$ $L_{5}$ идеала $T(b)$ в $N G_{2}(K)$. При $M \nsubseteq L_{2}$ любой элемент из $M$ по модулю централизатора $C(T(b))$ записывается в виде $x e_{b}+x^{\prime} e_{3 a+b}=\beta(x)$ для некоторых $x, x^{\prime} \in K$, причем $\beta(1) \in M$. Соотношения

$$
0=\beta(1) * \beta(x)= \pm\left(x^{\prime}-x d\right) e_{3 a+2 b}, \quad d=1^{\prime},
$$

дают равенство $x^{\prime}=x d$, так что $M$ совпадает с идеалом (1.1).

Допустим, что $2 K=0$. Пусть вначале в $M$ существует элемент $\gamma$ с простым углом $a$. Тогда произведение $M * e_{2 a+b}$ имеет угол $3 a+b$, и поэтому в $M$ нет элемента с углом $b$. Отсюда

$$
T(a) \supseteq M=C(M) \supseteq C(T(a))=L_{4}, \quad M \supset K\left(\gamma * e_{b}\right)+L_{4} \supset K e_{a+b} .
$$

Следовательно, элемент $\gamma \in M$ можно выбрать в виде $\gamma=e_{a}+c e_{2 a+b}$, где $c \in K$. Соотношения $0=e_{a+b} * \gamma=c e_{3 a+b}$ сразу же дают равенства $c=0$ и $M=K e_{a}+K e_{b+a}+L_{4}$.

Если идеал $M$ имеет элемент $\gamma$ с простым углом $b$, то аналогично находим включения

$$
T(b) \supseteq M=C(M) \supseteq C(T(b))=L_{5}, \quad M \supseteq L_{5}+K \alpha+K \gamma .
$$

Элементы $\alpha, \gamma$ здесь можно выбрать для некоторых $c, d \in K$ в виде

$$
\gamma=e_{b}+c e_{2 a+b}+d e_{3 a+b}, \quad \alpha=\gamma * e_{a}=e_{a+b}+c e_{3 a+b} .
$$

Легко видеть, что подалгебра с базой $\gamma, \gamma * e_{a}, e_{3 a+2 b}$ алгебры Ли $N G_{2}(K)$ является коммутативным идеалом при любом выборе $c, d \in K$. Таким образом, $M$ есть идеал вида (1.2).

В случае идеала $M$ с углом $a+b$ имеем

$$
T(a+b) \supseteq M=C(M) \supseteq C(T(a+b))=L_{4} .
$$

Поэтому $M$ содержит элемент $\alpha=e_{a+b}+d e_{2 a+b}$ для некоторого $d \in K$. Если $M$ содержит также элемент $\beta=x e_{a+b}+x^{\prime} e_{2 a+b}\left(x, x^{\prime} \in K\right)$, то из соотношения

$$
0=\alpha * \beta=\left(x^{\prime}+x d\right) e_{3 a+2 b}
$$

получаем равенство $x^{\prime}=x d$ и, следовательно, $M=K\left(e_{b+a}+d e_{b+2 a}\right)+L_{4}$. Остается заметить, что этот идеал при $d=0$ лежит в коммутативном идеале с простым углом $a$.

Это завершает доказательство теоремы.

Следствие 1. Наибольшая размерность коммутативных идеалов алгебры Ли $\mathrm{NG}_{2}(K)$ равна 4, когда $K$ - поле характеристики 2 или 3 , и равна 3 при $6 K=K$. Такие идеаль исчерпьвают идеаль $L_{2}$ и (1.1) при $3 K=0$, идеал $K e_{a}+K e_{b+a}+L_{4}$ nри $2 K=0$ и идеал $L_{3}$ при $6 K=K$.

Аналогичный подход мы применим в следующем разделе к алгебрам Ли $N \Phi(K)$ типа $F_{4}$. В общем случае для исследования гипотезы (A) эффективным оказывается использование метода, который А.И. Мальцев применял в исследованиях алгебры Ли $L_{\Phi}(C)$ и $N \Phi(C)$. Он называет подмножество корней $\Psi \subseteq \Phi$ коммутативным, если $r+s \notin \Phi \cup\{0\}$ для любых корней $r, s \in \Psi$ [2]. Е. П. Вдовин [8] обобщает это понятие. Подмножество $\Psi \subseteq \Phi$ он называет $p$-коммутативным для простого числа $p$, если в алгебре Ли $N \Phi(K)$ над любым полем $K$ характеристики $p$ имеем $e_{r} * e_{s}=0$ при всех $r, s \in \Psi$.

В [9] подмножество $\Psi \subseteq \Phi$ названо нормальным, если для любого корня $s \in \Psi$ выполняется включение $\{s\}^{+} \subseteq \Psi$. Если $p(\Phi):=\max \left\{(r, r) /(s, s) \mid r, s \in \Phi^{+}\right\}$и $p(\Phi) ! K=K$ (в частности, 
когда элементы 2 и 3 обратимы в поле $K$ ), понятия $p$-коммутативности и коммутативности совпадают.

Как и в [11, Lemma 5.3.1], далее используем регулярное упорядочение корней >; в этом случае из неравенства $\mathrm{ht}(r)>\mathrm{ht}(s)$ следует $r>s$. Первым углом произвольного ненулевого элемента $a \in N \Phi(K)$ назовем корень $s$, если в разложении $a=\sum_{r \in \Phi^{+}} \lambda_{r} e_{r}$ по базе, упорядоченной согласно возрастанию корней, первым ненулевым коэффициентом является $\lambda_{s}$. Множество первых углов всех элементов подмножества $M \subseteq N \Phi(K)$ обозначим через $\mathcal{L}_{1}(M)$.

Опираясь на методы работ [2;8], приходим к следующей теореме.

Теорема 2. Наибольшая размерность коммутативных подалгебр алгебры Ли $N \Phi(K)$ над полем K характеристики р равна наибольшему порядку р-коммутативных множеств корней в $\Phi$.

Д о к а з а т е л ь с т в о. Нам потребуются следующие две леммы.

Лемма 1. Если $M-$ коммутативнал подалгебра алгебры $N \Phi(K)$ над полем $K$ характеристики р, то множество корней $\mathcal{L}_{1}(M)$ является р-коммутативным.

Д о к а з а т е л ь с т в о. Если множество $\mathcal{L}_{1}(M)$ коммутативно, то оно $p$-коммутативно для любого простого числа $p$. Поэтому рассмотрим случай, когда существуют корни $s_{1}, t_{1} \in$ $\mathcal{L}_{1}(M)$ такие, что сумма $s_{1}+t_{1}$ является корнем. Выберем в $M$ элементы $\alpha$ и $\beta$ с первыми углами $s_{1}$ и $t_{1}$ соответственно:

$$
\alpha=a_{1} e_{s_{1}}+a_{2} e_{s_{2}}+\ldots+a_{n} e_{s_{n}}, \quad a_{1} \neq 0, \quad \beta=b_{1} e_{t_{1}}+b_{2} e_{t_{2}}+\ldots+b_{m} e_{t_{m}}, \quad b_{1} \neq 0 .
$$

Так как $s_{1}+t_{1}-$ наименьший из всевозможных корней $s_{i}+t_{j}$, то произведение

$$
\alpha * \beta=\sum_{i=1, \ldots, n, j=1, \ldots, m} a_{i} b_{j}\left(e_{s_{i}} * e_{t_{j}}\right)
$$

имеет при базисном элементе $e_{s_{1}+t_{1}}$ коэффициент $a_{1} b_{1} N_{s_{1}, t_{1}}$. В силу коммутативности подалгебры $M$ произведение $\alpha * \beta$ равно 0. Отсюда $e_{s_{1}} * e_{t_{1}}=N_{s_{1}, t_{1}} e_{s_{1}+t_{1}}=0$ и $p=\left|N_{s_{1}, t_{1}}\right|$ - простое число, равное $p=$ char $K$. Таким образом, множество корней $\mathcal{L}_{1}(M)$ является p-коммутативным.

Лемма доказана.

Лемма 2. Размерность подалгебры $M$ алгебры $N \Phi(K)$ равна порядку множсества $\mathcal{L}_{1}(M)$.

Д о к а з а т е л ь с т в о. Положим $n=\left|\mathcal{L}_{1}(M)\right| ;$ корни $\left\{r_{1}, \ldots, r_{n}\right\}$, составляющие $\mathcal{L}_{1}(M)$, располагаем в порядке возрастания. Выберем в $M$ элементы $\left\{a_{1}, \ldots, a_{n}\right\}$ так, что при любом $i$ корень $r_{i}$ есть первый угол элемента $a_{i}$. Нам достаточно показать, что элементы $a_{i}$ образуют базу в $M$.

Пусть $M_{i}(1 \leq i<n)$ есть подалгебра в $M$, которую линейно порождают $a_{i+1}, a_{i+2}, \ldots, a_{n}$, и $M_{n}=0$. Зафиксируем произвольный элемент $b \in M$. Согласно определению множества $\mathcal{L}_{1}(M)$ существует и единствен скаляр $\lambda_{1}$ такой, что первый угол элемента $b-\lambda_{1} a_{1}$ больше корня $r_{1}$. Более того, пользуясь индукцией, однозначно определяем скаляры $\lambda_{1}, \lambda_{2}, \ldots, \lambda_{n}$ такие, что

$$
b-\lambda_{1} a_{1}-\lambda_{2} a_{2} \cdots-\lambda_{i} a_{i} \in M_{i}, \quad i=1,2, \ldots, n .
$$

Следовательно, $b=\lambda_{1} a_{1}+\lambda_{2} a_{2}+\cdots+\lambda_{n} a_{n}$ и $\left\{a_{1}, \ldots, a_{n}\right\}$ - база в $M$.

Лемма доказана.

Теорема 2 непосредственно вытекает из лемм 1 и 2.

Поясним введенные понятия на примере идеалов $M$ из теоремы 1. Множество корней $\mathcal{L}_{1}(M)$ здесь нормально только при $M=L_{3}$ и $M=L_{2}$, причем в первом случае оно коммутативно, а во втором - 3-коммутативно, как и для идеала (1.1). Для остальных идеалов $M$ из теоремы 1 множество $\mathcal{L}_{1}(M)$ является $p$-коммутативным только при $p=2$. 


\section{2. Максимальные коммутативные идеалы алгебры $N F_{4}(K)$}

В этом разделе задача (Б) решена для типа $F_{4}$. Как и в [15], через $a b c d$ обозначаем корень $a \alpha_{1}+b \alpha_{2}+c \alpha_{3}+d \alpha_{4}$, где $\alpha_{1}, \alpha_{2}, \alpha_{3}, \alpha_{4}$ - простые корни системы корней типа $F_{4}$.

Перенесением теоремы 5.1 из [9] решение при $2 K=K$ устанавливает (см. [14]) следующую теорему.

Теорема 3. Максимальные коммутативные идеалы алгебры $N F_{4}(K)$ над полем $K=2 K$ исчерпывают идеал $T(1221)+T(0122)$ размерности 9 и идеалы $T(1221)+K\left(e_{1220}+d_{1122}\right)$, $d \in K$, размерности 8.

Следствие 2. Коммутативные идеалы наибольшей размерности алгебры $N F_{4}(K)$ над полем $K$ при сhar $K \neq 2$ исчерпьваются идеалом $T(1221)+T(0122)$ размерности 9.

Заметим, что при $p(\Phi) ! K=K$ описания больших абелевых подгрупп группы $U$ и коммутативных идеалов наибольшей размерности алгебры $N \Phi(K)$ в большинстве случаев совпадают. В общем случае это не так. Например, для случая $\Phi=F_{4}$, char $K=2$, все большие абелевы подгруппы группы $U$ лежат в $U_{3}$, а для соответствующей алгебры $N \Phi(K)$ существуют коммутативные идеалы наибольшей размерности с углом высоты 2.

Исследуем случай $2 K=0$. Выделим подалгебры

$$
\begin{gathered}
K\left(e_{1100}+c e_{1122}+d e_{1242}\right)+K\left(e_{1220}+c e_{1242}\right)+K e_{1110}+K e_{1111} \\
+K e_{1121}+K e_{1221}+K e_{1222}+K e_{1231}+K e_{1232}+K e_{1342}+K e_{2342}, \quad c, d \in K \\
K\left(e_{1110}+c e_{0120}+d e_{0121}\right)+K\left(e_{1111}+c e_{0121}\right) \\
+K e_{1120}+K e_{1220}+K e_{1121}+K e_{1221}+T(1231), \quad c \neq 0 \\
K\left(e_{1110}+c e_{0122}\right)+T(1111), \quad c \neq 0 ; \\
K e_{1110}+K\left(e_{1120}+c_{1} e_{1122}+c_{2} e_{1220}+c_{3} e_{1222}\right) \\
+K\left(e_{1220}+c_{1} e_{1222}\right)+K e_{1111}+K e_{1121}+K e_{1221}+T(1231), \quad c_{1}, c_{2}, c_{3} \in K \\
K e_{1110}+K\left(e_{1220}+c e_{1122}\right)+K e_{1111}+K e_{1121}+T(1221), \quad c \in K \\
K e_{0120}+K e_{1120}+K e_{0121}+K e_{1220}+K e_{1121}+K e_{1221}+T(1231) \\
K\left(e_{1120}+c e_{1222}\right)+K\left(c_{1} e_{1111}+c_{2} e_{0121}\right)+K e_{1220}+K e_{1121}+K e_{1221}+T(1231), \\
\left(c_{1}, c_{2}\right) \neq(0,0), \quad c \in K ; \\
K\left(c_{1} e_{1111}+c_{2} e_{0121}\right)+K\left(d_{1} e_{0122}+d_{2} e_{1220}\right)+T(1121), \quad\left(c_{1}, c_{2}\right) \neq(0,0), \quad\left(d_{1}, d_{2}\right) \neq(0,0) \\
K\left(c_{1} e_{1111}+c_{2} e_{0121}\right)+K\left(d_{1} e_{1122}+d_{2} e_{1220}\right)+K e_{1121}+T(1221), \\
\left(c_{1}, c_{2}\right) \neq(0,0), \quad\left(d_{1}, d_{2}\right) \neq(0,0) \\
T(0121) .
\end{gathered}
$$

Теорема 4. Максимальные коммутативные идеалы алгебры $N F_{4}(K)$ над полем $K$ характеристики 2 исчерпьваются идеалами (2.1)-(2.10). 
Д о к а з а т е л ь с т в о. Пусть $M$ - максимальный коммутативный идеал. Согласно [14] $M \subseteq T(1100)+T(0120)$. Под $r$-проекцией множества $M$ будем понимать множество $r$-проекций всех элементов из $M$.

Рассмотрим случай, когда $M \nsubseteq L_{3}$, т. е. $M$ имеет угол 1100 . Покажем, что в этом случае $M \subseteq T(1100)$. Если $M$ имеет угол 0120, то множество $M * e_{0001} \subseteq M$ имеет угол 0121 и содержится в $T(1111)+T(0121)$. Множество

$$
\left(M * e_{0010}\right) * e_{0001} \subseteq M
$$

имеет единственный угол 1111, следовательно, множество

$$
\left(M * e_{0001}\right) *\left(\left(M * e_{0010}\right) * e_{0001}\right)
$$

имеет ненулевую 1231-проекцию, что противоречит коммутативности $M$. Таким образом, $M \subseteq$ $T(1100)+T(0121)$.

Аналогично, если $M$ имеет угол 0121, то множество

$$
M *\left(\left(M * e_{0010}\right) * e_{0001}\right)
$$

имеет ненулевую 1232 -проекцию, что противоречит коммутативности $M$. Таким образом, $M \subseteq$ $T(1100)+T(0122)$. Наконец, если $M$ имет угол 0122 , то множество $\left(M * e_{0010}\right) * M$ имеет ненулевую 1232-проекцию. Таким образом, $M \subseteq T(1100)$.

Кроме того, 1120-проекция множества $M$ нулевая, так как в противном случае множество $M *\left(M * e_{0122}\right)$ будет иметь ненулевую 1342 -проекцию, что противоречит коммутативности $M$.

Заметим, что идеал

$$
I=K e_{1110}+K e_{1111}+K e_{1121}+K e_{1221}+K e_{1222}+K e_{1231}+K e_{1232}+K e_{1342}+K e_{2342}
$$

централизует $M$, следовательно, содержится в $M$ в силу его максимальности. Запишем произвольный элемент $a \in M$ в виде

$$
a=a_{1100} e_{1100}+a_{1220} e_{1220}+a_{1122} e_{1122}+a_{1242} e_{1242} \bmod I .
$$

Так как $a * x e_{0120}=x a_{1100} e_{1220}+x a_{1122} e_{1242} \bmod I, x \in K$, то из соотношения $M *\left(M * e_{0120}\right)=0$ получаем, что $a_{1122}=c a_{1100}$ для некоторого $c \in K$, т. е.

$$
M \supseteq K\left(e_{1220}+c e_{1242}\right) .
$$

Рассмотрим произвольный элемент $b \in M$ с углом 1220. Запишем его в виде

$$
b=b_{1220} e_{1220}+b_{1122} e_{1122}+b_{1242} e_{1242} \bmod I .
$$

Так как $a * b=\left(a_{1100} b_{1242}+c a_{1100} b_{1220}\right) e_{2342}=0$, то $b_{1242}=c b_{1220}$. Тогда $b_{1122}=0$, поскольку в противном случае, учитывая соотношение (2.11), получаем $M \supseteq K e_{1122}$, но $K e_{1122} * K\left(e_{1220}+\right.$ $\left.c e_{1242}\right) \neq 0$. Таким образом, $M$ совпадает с идеалом $(2.1)$.

Рассмотрим случай, когда $M \subseteq L_{3}$ и $M$ имеет угол высоты 3. Пусть $M$ имеет угол 1110 . Тогда идеал

$$
K e_{1121}+K e_{1221}+T(1231)
$$

централизует $M$, следовательно, содержится в $M$ в силу его максимальности.

Рассмотрим элемент $a \in M$ вида

$$
a=a_{1110} e_{1110}+a_{0120} e_{0120} \bmod L_{4}, \quad a_{1110} \neq 0 .
$$

Тогда

$$
a * e_{0001}=a_{1110} e_{1111}+a_{0120} e_{0121} \quad \bmod K e_{1121}+K e_{1221}+T(1231) .
$$


Если $M$ содержит элемент $b=b_{0120} e_{0120} \bmod L_{4}, b_{0120} \neq 0$, то произведение $\left(a * e_{0001}\right) * b$ имеет ненулевую 1231-проекцию. Следовательно, $a_{0120}=c a_{1110}$ для некоторого $c \in K$. Кроме того, в силу коммутативности $M$ имеем $a_{0121}=d a_{1110}$ для некоторого $d \in K$.

Предположим, что $M$ имеет еще угол 0120. Тогда при ненулевой 0122-проекции множества $M$ множество $M *\left(M * e_{1000}\right)$ имеет ненулевую 1242 -проекцию, что противоречит коммутативности $M$. Таким образом, 0122-проекция множества $M$ нулевая. Следовательно,

$$
M * K e_{1000}=K e_{1120} \bmod K e_{1121}+K e_{1221}+T(1231),
$$

откуда в силу коммутативности 1222-проекция (а следовательно, и 1122-проекция) в $M$ нулевые. Таким образом, $M$ совпадает с идеалом (2.2).

Если 0120-проекция в $M$ нулевая, то

$$
M \supseteq M * K e_{0001} \supseteq K e_{1111} \bmod K e_{1121}+K e_{1221}+T(1231),
$$

откуда в силу коммутативности 0121-проекция в $M$ нулевая. При ненулевой 0122-проекции $M$ совпадает с идеалом (2.3), а при нулевой - с одним из идеалов (2.4) или (2.5).

Если $M$ содержится в $T(0120)+L_{4}$ и имеет угол 0120 , легко показать, что $M$ совпадает с идеалом (2.6).

Пусть $M \subseteq L_{4}$. Если $M$ имеет угол 1120 , то множество $M * e_{0100} \subseteq M$ содержит элемент с единственным углом 1220 , следовательно, 1122 -проекция в $M$ нулевая и $M$ совпадает с идеалом (2.7).

Наконец, если $M \subseteq T(1111)+T(0121)+T(1220)$, то $M$ совпадает с одним из идеалов $(2.8)$, (2.9) или (2.10).

Теорема доказана.

Следствие 3. Коммутативные идеаль наибольшей размерности алгебры $N F_{4}(K)$ над полем K характеристики 2 исчерпьваются идеалами (2.1)-(2.6), (2.8) и (2.10) размерности 11.

\section{3. Наибольшая размерность коммутативных подалгебр алгебры $N \Phi(K)$}

Далее для исследования гипотезы (А) ограничимся описанием коммутативных идеалов наибольшей размерности алгебры $N \Phi(K)$.

С этой целью в [13] используется специальное матричное представление из [16] алгебры Ли $N \Phi(K)$ классических типов. Для типа $A_{n-1}$ ее ассоциируют с алгеброй $N T_{n}(K)$ нильтреугольных $n \times n$-матриц над $K$. Обычные матричные единицы $e_{i j}$ здесь составляют базу Шевалле $\left\{e_{r} \mid r \in \Phi^{+}, e_{r}=e_{i j}\right\}$ алгебры Ли $N \Phi(K)$ после соответствующей нумерации корней $r=r_{i j}$ $(1 \leq j<i \leq n)$.

Алгебры Ли $N \Phi(K)$ классических типов $B_{n}, C_{n}$ и $D_{n}$ заданы в [16] базисом из "матричных единиц" $e_{i v}$ с ограничениями $i \leq n$ и, соответственно,

$$
-i<v<i, \quad-i \leq v<i(v \neq 0), \quad 1 \leq|v|<i .
$$

При $e_{r}=e_{i v}$ полагаем $r=p_{i v}$ и $T(r)=T_{i v}$. Помимо сумм $p_{i j}+p_{j v}=p_{i v}$ (аналогично типу $\left.A_{n-1}\right)$ к суммам двух корней, являющимся корнем, здесь относят еще $p_{k v}+p_{m,-v}=p_{k,-m}$ при $k>m>|v|$, а для типа $C_{n}$ - также при $k=m>|v|$. Структурные константы выписаны по теореме Шевалле о базисе [16, лемма 2].

В [13] приведена таблица (см. табл. 1) и установлена следующая теорема.

Теорема 5. Коммутативные идеалы наибольшей размерности алгебры $N \Phi(K)$ классического типа над полем $K$ исчерпьваются идеалами из табл. 1. 
Т а б ли и ц 1

Коммутативные идеалы наибольшей размерности алгебры $N \Phi(K)$ классических типов

\begin{tabular}{|c|c|c|}
\hline Тип $N \Phi(K)$ & $\begin{array}{c}\text { Коммутативные идеалы } \\
\text { наибольшей размерности }\end{array}$ & $\begin{array}{l}\text { Наибольшая } \\
\text { размерность }\end{array}$ \\
\hline $\begin{array}{l}A_{2 n-1} \\
A_{2 n}, \quad n>1 \\
A_{2}\end{array}$ & $\begin{array}{l}T_{n+1, n} \\
T_{n+1, n}, T_{n+2, n+1} \\
L_{2}+K \alpha, \quad \alpha \notin L_{2}\end{array}$ & $\begin{array}{c}n^{2} \\
n(n+1) \\
2\end{array}$ \\
\hline $\begin{array}{l}B_{n}, 2 K=K ; n=3 \\
n>4 \\
n=4\end{array}$ & $\begin{array}{l}T_{n n-1} \\
T_{2,-1}+T_{n 0} \\
T_{2,-1}+T_{n 0}, T_{n n-1}\end{array}$ & $\begin{array}{c}5 \\
\left(\begin{array}{l}n \\
2\end{array}\right)+1\end{array}$ \\
\hline$B_{n}, 2 K=0 ; n>2$ & $T_{10}$ & $\left(\begin{array}{c}n+1 \\
2\end{array}\right)$ \\
\hline$B_{2}=C_{2}, 2 K=0$ & $L_{2}+K \alpha, \quad \alpha \notin L_{2}$ & 3 \\
\hline $\begin{array}{l}C_{n} ; n>2 \text { или } \\
2 K=K, n=2\end{array}$ & $T_{1,-1}$ & $\left(\begin{array}{c}n+1 \\
2\end{array}\right)$ \\
\hline$D_{n}, \quad n>4$ & $\begin{array}{l}T_{3,-2}+\sum_{m=2}^{n} K\left(a e_{m 1}+b e_{m,-1}\right), \\
(a, b) \neq(0,0) \text { при } 2 K=0 \text { и } \\
(a, b)=(1,0) \text { или }(0,1) \text { при } 2 K=K\end{array}$ & $\left(\begin{array}{l}n \\
2\end{array}\right)$ \\
\hline$D_{4}, \quad 2 K=K$ & $T\left(p_{2,-1}\right), T\left(p_{21}\right), T\left(p_{43}\right)$ & $\left(\begin{array}{l}4 \\
2\end{array}\right)$ \\
\hline$D_{4}, 2 K=0$ & $\begin{array}{l}T_{3,-2}+\sum_{m=2}^{4} K\left(e_{m 1}+a e_{m,-1}\right), T_{2,-1} \\
\text { и их автоморфные образы }\end{array}$ & $\left(\begin{array}{l}4 \\
2\end{array}\right)$ \\
\hline
\end{tabular}

Т а б ли и а 2

Коммутативные идеалы наибольшей размерности алгебры $N \Phi(K)$ исключительных типов

\begin{tabular}{|l|l|c|}
\hline Тип $N \Phi(K)$ & \multicolumn{1}{|c|}{$\begin{array}{c}\text { Коммутативные идеалы } \\
\text { наибольшей размерности }\end{array}$} & $\begin{array}{c}\text { Наибольшая } \\
\text { размерность }\end{array}$ \\
\hline$E_{6}$ & $\begin{array}{l}\left.T_{(}\left(\alpha_{1}\right), T_{(} \alpha_{6}\right) \\
E_{7}\end{array}$ & 16 \\
$E_{8}$ & $T\left(\alpha_{7}\right)$ & 27 \\
\hline$F_{4}, 2 K=K$ & $T(1221)+T(0122)$ & 36 \\
$F_{4}, 2 K=0$ & идеалы $(2.1)-(2.6),(2.8)$ и $(2.10)$ & 9 \\
\hline$G_{2}, 6 K=K$ & $L_{3}$ & 3 \\
$G_{2}, 2 K=0$ & $K e_{a}+K e_{b+a}+L_{4}$ & 4 \\
$G_{2}, 3 K=0$ & $L_{2}, K\left(e_{b}+d e_{b+3 a}\right)+K e_{b+a}+K e_{b+2 a}+L_{5}(d \in K)$ & 4 \\
\hline
\end{tabular}

В следующей теореме приводится описание коммутативных идеалов наибольшей размерности для оставшегося случая $\Phi=E_{n}, n=6,7,8$.

Теорема 6. Коммутативные идеаль наибольшей размерности алгебры $N E_{6}(K)$ исчерпываются идеалами $T\left(\alpha_{1}\right), T\left(\alpha_{6}\right)$ размерности 16 , алгебры $N E_{7}(K)-$ идеалом $T\left(\alpha_{7}\right)$ размерности 27 , алгебры $N E_{8}(K)-$ идеалом $T\left(\alpha_{1}+2 \alpha_{2}+2 \alpha_{3}+3 \alpha_{4}+2 \alpha_{5}+\alpha_{6}\right)$ размерности 36. 
Д о к а з а т е л ь с т в о. Базисные корни систем корней типа $E_{n}$ обозначим через $\alpha_{i}$, $i=1, \ldots, n,[15]$. Согласно [2] каждая система корней типа $E_{n}$ имеет нормальное максимальное коммутативное подмножество корней. Для типа $E_{6}$ такими подмножествами являются $\left\{\alpha_{1}\right\}^{+}$ и $\left\{\alpha_{6}\right\}^{+}$порядка 16 , для типа $E_{7}$ это $\left\{\alpha_{7}\right\}^{+}$порядка 27 , а для типа $E_{8}$ это $\left\{\alpha_{1}+2 \alpha_{2}+2 \alpha_{3}+\right.$ $\left.3 \alpha_{4}+2 \alpha_{5}+\alpha_{6}\right\}^{+}$порядка 36 . Им можно сопоставить коммутативные идеалы соответствующих размерностей: $T\left(\alpha_{1}\right), T\left(\alpha_{6}\right), T\left(\alpha_{7}\right)$ и $T\left(\alpha_{1}+2 \alpha_{2}+2 \alpha_{3}+3 \alpha_{4}+2 \alpha_{5}+\alpha_{6}\right)$.

Покажем, что приведенными выше идеалами исчерпываются все коммутативные идеалы наибольшей размерности. Пусть $M$ - произвольный коммутативный идеал наибольшей размерности подалгебры $N \Phi(K)$ типа $\Phi=E_{n}, \rho$ - максимальный корень системы $E_{n}^{+}$. В случае $n=6$ и $\mathcal{L}_{1}(M)=\left\{\alpha_{1}\right\}^{+}$в силу соотношения

$$
M \supseteq\left(\left(M * e_{\alpha_{3}}\right) * e_{\alpha_{4}}\right) * K e_{\alpha_{2}}=K e_{\alpha_{1}+\alpha_{2}+\alpha_{3}+\alpha_{4}} \quad \bmod K e_{\rho}
$$

и коммутативности $M$ получаем, что $\left(\alpha_{2}+\alpha_{3}+2 \alpha_{4}+2 \alpha_{5}+\alpha_{6}\right)$-проекция в $M$ нулевая, и поэтому $M \subseteq T\left(\alpha_{1}\right)$. Случай $\mathcal{L}_{1}(M)=\left\{\alpha_{6}\right\}^{+}$рассматривается аналогично. Для типа $E_{7}$ из соотношения

$$
M \supseteq\left(\left(\left(\left(M * e_{\alpha_{6}}\right) * e_{\alpha_{5}}\right) * e_{\alpha_{4}}\right) * e_{\alpha_{3}}\right) * K e_{\alpha_{1}}=K e_{\alpha_{1}+\alpha_{3}+\alpha_{4}+\alpha_{5}+\alpha_{6}+\alpha_{7}} \quad \bmod K e_{\rho}
$$

получаем, что $\left(\alpha_{1}+2 \alpha_{2}+2 \alpha_{3}+3 \alpha_{4}+2 \alpha_{5}+\alpha_{6}\right)$-проекция в $M$ является нулевой, и поэтому $M \subseteq T\left(\alpha_{7}\right)$. Для типа $E_{8}$ из включения

$$
M \supseteq\left(M * e_{\alpha_{7}}\right) * K e_{\alpha_{8}}=K e_{\alpha_{1}+2 \alpha_{2}+2 \alpha_{3}+3 \alpha_{4}+2 \alpha_{5}+\alpha_{6}+\alpha_{7}+\alpha_{8}} \quad \bmod K e_{\rho}
$$

вытекает, что $\left(\alpha_{1}+\alpha_{2}+2 \alpha_{3}+3 \alpha_{4}+3 \alpha_{5}+3 \alpha_{6}+2 \alpha_{7}+\alpha_{8}\right)$-проекция в $M$ нулевая и $M \subseteq$ $T\left(\alpha_{1}+2 \alpha_{2}+2 \alpha_{3}+3 \alpha_{4}+2 \alpha_{5}+\alpha_{6}\right)$. Теорема доказана.

Резюмируем полученные результаты в табл. 2.

Гипотезу (А) подтверждает следующая теорема.

Теорема 7. Всякий коммутативный идеал наибольшей размерности алгебры $N \Phi(K)$ является ее коммутативной подалгеброй наибольшей размерности. Коммутативные идеаль наибольшей размерности алгебры $N \Phi(K)$ над полем $K$ исчерпываются идеалами из табл. 1 и табл. 2.

Д о к а з а т е л ь с т в о. Согласно теореме 2 для проверки истинности гипотезы (А) для алгебры $N \Phi(K)$ над полем $K$ характеристики $p$ достаточно сравнить наибольшую размерность коммутативных идеалов этой алгебры и наибольший порядок $p$-коммутативных множеств корней в $\Phi$.

Максимальный порядок коммутативных множеств корней системы типа $G_{2}$ равен 3 [2], a 2- и 3-коммутативных множеств корней -4 [8], что подтверждает гипотезу при $\Phi=G_{2}$. Максимальный порядок коммутативных множеств корней системы типа $F_{4}$ равен 9 [2], а 2коммутативных множеств корней -11 [8]. Таким образом, для $\Phi=F_{4}$ справедливость гипотезы также доказана. Теорема 6 подтверждает гипотезу для типов $E_{n}$, с учетом описания максимальных коммутативных множеств корней для соответствующих типов из [2]. Остается заметить, что для классических типов подтверждение гипотезы вытекает из [9;13].

Теорема доказана.

Для конечных групп $G$ лиева типа почти всегда большие абелевы подгруппы группы $U$ являются $G$-сопряженными с нормальными подгруппами группы $U$ [9, Theorem 6.5]. Аналогично, для решения обобщенной редукционной задачи достаточно получить список коммутативных подалгебр наибольшей размерности с точностью до автоморфизмов алгебры Шевалле (см. также [17]). 


\section{СПИСОК ЛИТЕРАТУРЫ}

1. Schur I. Zur theorie der vertauschbaren matrizen // J. reine und angew. Math. 1905. Vol. 130. P. 66-76.

2. Мальцев А.И. Коммутативные подалгебры полупростых алгебр Ли // Изв. АН СССР. Сер. математическая. 1945. Т. 9, № 4. С. 291-300.

3. Barry M. J.J. Large Abelian subgroups of Chevalley groups // J. Austral. Math. Soc. Ser. A. 1979. Vol. 27, no. 1. P. 59-87. doi: 10.1017/S1446788700016645.

4. Wong W.J. Abelian unipotent subgroups of finite orthogonal groups // J. Austral. Math. Soc., Ser. A. 1982. Vol. 32, no. 2. P. 223-245. doi: 10.1017/S1446788700024575.

5. Wong W.J. Abelian unipotent subgroups of finite unitary and symplectic groups // J. Austral. Math. Soc., Ser. A. 1982. Vol. 33, no. 2. P. 331-344. doi: 10.1017/S1446788700018759.

6. Кондратьев А.С. Подгруппы конечных групп Шевалле // Успехи мат. наук. 1986. Т. 41, № 1 (247). С. 57-96.

7. Вдовин Е.П. Максимальные порядки абелевых подгрупп в конечных группах Шевалле // Мат. заметки. 2000. Т. 68, вып. 1. С. 53-76.

8. Вдовин Е.П. Большие абелевы унипотентные подгруппы конечных групп Шевалле // Алгебра и логика. 2001. Т. 40, № 5. С. 523-544.

9. Levchuk V.M., Suleimanova G.S. Extremal and maximal normal abelian subgroups of a maximal unipotent subgroup in groups of Lie type // J. Algebra. 2012. Vol. 349, iss. 1. P. 98-116. doi: $10.1016 /$ j.jalgebra.2011.10.025.

10. Levchuk V.M., Suleimanova G.S. Thompson subgroups and large abelian unipotent subgroups of Lie-type groups // J. Siberian Federal University. Math. \& Physics. 2013. Vol. 6, no. 1. P. 64-74.

11. Carter R. Simple groups of Lie type. New York: Wiley and Sons, 1972. 332 p. ISBN 0-471-13735-9 .

12. Levchuk V.M., Suleimanova G.S. A problem of large abelian subgroups and the generalized Mal'cev problem // Proc. XII Intern. Conf. "Algebra and number theory". Тула: Изд-во Тульского гос. пед. ун-та, 2014. С. 108-111. ISBN: 978-5-87954-874-7,

13. Levchuk V.M., Suleimanova G.S. The generalized Mal'cev problem on abelian subalgebras of the Chevalley algebras // Lobachevskii J. Math. 2015. Vol. 86. No. 4. P. 384-388. doi: 10.1134/S1995080215040083.

14. Кравцова Е.А. Максимальные коммутативные идеалы подалгебры $\mathrm{N} \Phi(\mathrm{K})$ алгебр Ли типа F4 // Молодежь и наука: сб. материалов Х Всерос. науч.-техн. конф. студентов, аспирантов и молодых ученых. Красноярск: Сиб. федеральный ун-т, 2014. ISBN: 978-5-7638-3102-3 .

15. Бурбаки Н. Группы и алгебры Ли (гл. IV-VI). М.: Мир, 1976. 332 с.

16. Левчук В. М. Автоморфизмы унипотентных подгрупп групп Шевалле // Алгебра и логика. 1990. T. 29, № 3. С. 315-338.

17. Левчук В.М., Литаврин А.В. Гиперцентральные автоморфизмы нильтреугольных подалгебр алгебр Шевалле // Сиб. электрон. мат. изв. 2016. Т. 13. С. 467-477.

Кириллова Евгения Алексеевна

Поступила 10.06.2018

аспирант

Институт математики и фундаментальной информатики

Сибирский федеральный университет,

г. Красноярск

e-mail: kea92@bk.ru

Сулейманова Галина Сафиуллановна

д-р физ.-мат. наук, доцент

профессор кафедры ПИМиЕД

Хакасский технический институт

(филиал Сибирского федерального университета),

г. Абакан

e-mail: suleymanova@list.ru 


\section{REFERENCES}

1. Schur I. Zur theorie der vertauschbaren matrizen. J. reine und angew. Math., 1905, vol. 130, pp. 66-76.

2. Mal'tsev A.I. Commutative subalgebras of semi-simple Lie algebras. Izvestia Akad. Nauk SSSR, Ser. Mat., 1945, vol. 9, no. 4, pp. 291-300 (in Russian).

3. Barry M.J.J. Large Abelian subgroups of Chevalley groups. J. Austral. Math. Soc. Ser. A, 1979, vol. 27, no. 1, pp. 59-87. doi: 10.1017/S1446788700016645.

4. Wong W.J. Abelian unipotent subgroups of finite orthogonal groups. J. Austral. Math. Soc., Ser. A, 1982, vol. 32, no. 2, pp. 223-245. doi: 10.1017/S1446788700024575.

5. Wong W.J. Abelian unipotent subgroups of finite unitary and symplectic groups. J. Austral. Math. Soc., Ser. A, 1982, vol. 33, no. 2, pp. 331-344. doi: 10.1017/S1446788700018759 .

6. Kondrat'ev A.S. Subgroups of finite Chevalley groups. Russian Math. Surveys, 1986, vol. 41, no. 1, pp. 65-118. doi: 10.1070/RM1986v041n01ABEH003203.

7. Vdovin E.P. Maximal orders of Abelian subgroups in finite Chevalley groups. Math. Notes, 2001, vol. 69, no. 3-4, pp. 475-498. doi: 10.1023/A:1010256129959.

8. Vdovin E.P. Large Abelian unipotent subgroups of finite Chevalley groups. Algebra and Logic, 2001, vol. 40, no. 5, pp. 292-305. doi: 10.1023/A:1012549701336.

9. Levchuk V.M., Suleimanova G.S. Extremal and maximal normal abelian subgroups of a maximal unipotent subgroup in groups of Lie type. J. Algebra, 2012, vol. 349, no. 1, pp. 98-116. doi: $10.1016 /$ j.jalgebra.2011.10.025.

10. Levchuk V.M., Suleimanova G.S. Thompson subgroups and large abelian unipotent subgroups of Lietype groups. J. Siberian Federal. University. Math. \& Physics, 2013, vol. 6, no. 1, pp. 63-73.

11. Carter R. Simple groups of Lie type. N Y: Wiley and Sons, 1972. 332 p. ISBN 0-471-13735-9 .

12. Levchuk V.M., Suleimanova G.S. A problem of large Abelian subgroups and the generalized Mal'cev problem. Proceed. XII Intern. conf. "Algebra and number theory". Tula: Tul'sk. Gos. Ped. Univ. Publ., 2014, pp. 108-111. ISBN: 978-5-87954-874-7.

13. Levchuk V. M., Suleimanova G. S. The generalized Mal'cev problem on Abelian subalgebras of the Chevalley algebras. Lobachevskii J. Math., 2015, vol. 36, no. 4, pp. 384-388.

doi: 10.1134/S1995080215040083.

14. Kravtsova E.A. Maximal commutative ideals of subalgebra NF(K) of Lie algebras of type F4. Proc. 10th All-Russian Sci. Tech. Conf. "Molodezh'i nauka" (Youth and Science). Krasnoyarsk: Sib Fed. Univ. Publ., 2014 (in Russian). ISBN: 978-5-7638-3102-3.

15. Bourbaki N. Groupes et algebres de Lie (Chapt. IV-VI). Paris: Hermann, 1968, 282 p. doi: 10.1007/9783-540-34491-9. Translated to Russian under the title Gruppy i algebry Li (glavy IV-VI). Moscow: Mir Publ., 1976, 332 p.

16. Levchuk V.M. Automorphisms of unipotent subgroups in Chevalley groups. Algebra and Logic, 1990, vol. 29, no. 3, pp. 211-224. doi: 10.1007/BF01979936.

17. Levchuk V.M., Litavrin A.V. Hypercentral automorphisms of nil-triangular subalgebras in Chevalley algebras. Sib. Elektron. Mat. Izv., 2016, vol. 13, pp. 467-477 (in Russian). doi: $10.17377 /$ semi.2016.13.040 .

The paper was received by the Editorial Office on Juny 10, 2018.

Funding Agency: This work was supported by the Russian Foundation for Basic Research (project no. 16-01-00707).

Evgeniya Alekseevna Kirillova, doctoral student, Siberian Federal University, Krasnoyarsk, 660041 Russia, e-mail: kea92@bk.ru.

Galina Safiullanovna Suleimanova, Dr. Phys.-Math. Sci., Khakas Technical Institute - Branch of Siberian Federal University, Abakan, 655017 Russia, e-mail: suleymanova@list.ru . 